\title{
On some subclasses $k$-uniformly Janowski starlike and convex functions associated with $t$-symmetric points
}

\author{
Khalida Inayat Noor $^{1}$ (D), Nasir Khan ${ }^{2}$ (D), Muhammad Arif ${ }^{3}$ (D), Janusz Sokół ${ }^{* 4}$ (D) \\ ${ }^{1}$ Department of Mathematics COMSATS Institute of Information Technology, Park Road, Islamabad, \\ Pakistan \\ ${ }^{2}$ Department of Mathematics FATA University F.R. Kohat, Pakistan \\ ${ }^{3}$ Department of Mathematics, Abdul Wali Khan University Mardan, Mardan 23200, Pakistan \\ ${ }^{4}$ Faculty of Mathematics and Natural Sciences, University of Rzeszów, ul. Prof. Pigonia 1, 35-310 \\ Rzeszów, Poland
}

\begin{abstract}
In this paper, we define new subclasses of $k$-uniformly Janowski starlike and $k$-uniformly Janowski convex functions associated with $t$-symmetric points. The integral representations, convolution properties and coefficient bounds for these classes are studied.
\end{abstract}

Mathematics Subject Classification (2010). 30C45, 30C50

Keywords. subordination, convolution, $t$-symmetric points

\section{Introduction}

Let $\mathcal{A}$ denote the class of functions $f(z)$ of the form

$$
f(z)=z+\sum_{n=2}^{\infty} a_{n} z^{n}
$$

which are analytic in the open unit disk $U=\{z: z \in \mathbb{C}$ and $|z|<1\}$. Furthermore, $\mathcal{S}$ represents the class of all functions in $\mathcal{A}$ which are univalent in $U$.

Sakaguchi [10], introduced a class $\mathcal{S}_{s}^{*}$ of functions starlike with respect to symmetric points, it consists of functions $f(z) \in \mathcal{S}$, satisfying the inequality

$$
\mathfrak{R e}\left\{\frac{f^{\prime}(z)}{f(z)-f(-z)}\right\}>0, \quad(z \in U) .
$$

Following him, many authors studied this class and its subclasses see $[1,9,12,14]$.

Motivated by $\mathcal{S}_{s}^{*}$, we can easily obtain the following class $C_{s}$ of functions convex with respect to symmetric points. Let $C_{s}$ denote the class of functions in $\mathcal{S}$, satisfying the inequality

$$
\mathfrak{R e}\left\{\frac{\left(z f^{\prime}(z)\right)^{\prime}}{f^{\prime}(z)-f^{\prime}(-z)}\right\}>0, \quad(z \in U) .
$$

\footnotetext{
*Corresponding Author.

Email addresses: khalidanoor@hotmail.com (K.I. Noor), dr.nasirkhan@fu.edu.pk (N. Khan), marifmaths@awkum.edu.pk (M. Arif), jsokol@ur.edu.pl (J. Sokół)

Received: 15.04.2015; Accepted: 18.03.2019
} 
In [2], Chand and Singh considered a class $\mathcal{S}_{s}^{t}$ of functions starlike with respect to $t$-symmetric points, which consists of functions $f(z) \in \mathcal{S}$, satisfying the inequality

$$
\mathfrak{R e}\left\{\frac{z f^{\prime}(z)}{f_{t}(z)}\right\}>0, \quad(z \in U),
$$

where

$$
f_{t}(z)=\frac{1}{t} \sum_{\mu=0}^{t-1} \epsilon^{-\mu} f\left(\epsilon^{\mu} z\right), \quad\left(\epsilon^{t}=1: t \in \mathbb{N}\right) .
$$

From (1.1) equation (1.3) we can rewrite as

$$
\begin{aligned}
f_{t}(z) & =\frac{1}{t} \sum_{\mu=0}^{t-1} \epsilon^{-\mu} f\left(\epsilon^{\mu} z\right)=\frac{1}{t} \sum_{\mu=0}^{t-1} \epsilon^{-\mu}\left[\epsilon^{\mu} z+\sum_{n=2}^{\infty} a_{n}\left(\epsilon^{\mu} z\right)^{n}\right] \\
& =z+\sum_{n=2}^{\infty} b_{n} a_{n} z^{n}
\end{aligned}
$$

where

$$
b_{n}=\frac{1}{t} \sum_{\mu=0}^{t-1} \epsilon^{(n-1) \mu}= \begin{cases}1, & n=l t+1, \\ 0, & n \neq l t+1,\end{cases}
$$

where $l, t \in \mathbb{N} ; n \geq 2 ; \epsilon^{t}=1$.

Notice that

$$
\begin{aligned}
& f_{t}\left(\epsilon^{\mu} z\right)=\epsilon^{\mu} f_{t}(z) \\
& f_{t}^{\prime}\left(\epsilon^{\mu} z\right)=f_{t}(z)=\frac{1}{t} \sum_{\mu=0}^{t-1} f^{\prime}\left(\epsilon^{\mu} z\right), \quad(z \in U) .
\end{aligned}
$$

Definition 1.1. For $f(z) \in \mathcal{A}$, given by $(1.1)$ and $g(z) \in \mathcal{A}$ of the form

$$
g(z)=z+\sum_{n=2}^{\infty} c_{n} z^{n}, \quad(z \in U)
$$

the Hadmard product (or convolution) of $f(z)$ and $g(z)$ is given by

$$
(f * g)(z)=z+\sum_{n=2}^{\infty} a_{n} c_{n} z^{n}=(g * f)(z), \quad(z \in U) .
$$

For two functions $f(z)$ and $g(z)$ analytic in $U$, we say that $f(z)$ is subordinate to $g(z)$, denoted by $f \prec g$ or $f(z) \prec g(z)$, if there exists an analytic function $w(z)$ with $|w(z)|<|z|$ such that $f(z)=g(w(z))$. If $g(z)$ is univalent in $U$ then $f(z) \prec g(z)$ if and only if $f(0)=g(0)$ and $f(U) \subset g(U)$. The idea of subordination was widely presented by Miller and Mocanu [7].

Definition 1.2. A function $p(z)$ is said to be in the class $P[A, B],-1 \leq B<A \leq 1$, if it is analytic in $U$ with $p(0)=1$ and

$$
p(z) \prec \frac{1+A z}{1+B z}, \quad(z \in U) .
$$

Geometrically, if a function $p$ belongs to $P[A, B]$, then it maps the open unit disc $U$ onto the disk

$$
\Omega[A, B]=\left\{w:\left|w-\frac{1-A B}{1-B^{2}}\right|<\frac{A-B}{1-B^{2}}\right\} .
$$

The class $P[A, B]$ is connected with the class $P$ of functions with positive real part by the relation

$$
p(z) \in P, \quad \text { if and only if } \quad \frac{(A+1) p(z)-(A-1)}{(B+1) p(z)-(B-1)} \in P[A, B] .
$$


This class $P[A, B]$ was presented by Janowski [3] and explored by a few creators. Kanas and Wiśniowska $[4,5]$ presented and examined the class $k-S T$ of $k$-starlike functions and the related class $k-U C V$ of $k$-uniformly convex functions. These classes were characterized subject to the conic region $\Omega_{k}, k \geq 0$, as

$$
\Omega_{k}=\left\{u+i v: u>k \sqrt{(u-1)^{2}+v^{2}}\right\} .
$$

This domain represents the right half plane, a parabola, a hyperbola and an ellipse for $k=0, k=1,0<k<1$ and $k>1$ respectively. The functions such that $p_{k}(U)=\Omega_{k}$ are

$$
p_{k}(z)= \begin{cases}\frac{1+z}{1-z}, & k=0, \\ 1+\frac{2}{\pi^{2}}\left(\log \frac{1+\sqrt{z}}{1-\sqrt{z}}\right)^{2}, & k=1, \\ 1+\frac{2}{1-k^{2}} \sinh ^{2}\left\{\left(\frac{2}{\pi} \arccos k\right) \operatorname{arctanh} \sqrt{z}\right\}, & 0<k<1 \\ 1+\frac{2}{k^{2}-1} \sin \left(\frac{\pi}{2 R(t)} \int_{0}^{\frac{u(z)}{\sqrt{t}}} \frac{d x}{\sqrt{1-x^{2}} \sqrt{1-(t x)^{2}}}\right)+\frac{1}{k^{2}-1}, & k>1\end{cases}
$$

where

$$
u(z)=\frac{z-\sqrt{t}}{1-\sqrt{t x}}, \quad(z \in U)
$$

and $t \in(0,1)$ and $z$ is chosen such that $k=\cosh \left(\frac{\pi R^{\prime}(t)}{4 R(t)}\right)$. Here $R(t)$ is Legendre's complete elliptic integral of first kind and $R^{\prime}(t)$ is the complementary integral of $R(t)$.

Following are the definitions of classes $k-S T$ and $k-U C V$.

Definition 1.3. A function $f(z) \in \mathcal{A}$ is said to be in the class $k-S T, k \geq 0$, if and only if,

$$
\frac{z f^{\prime}(z)}{f(z)} \prec p_{k}(z), \quad(z \in U) .
$$

Definition 1.4. A function $f(z) \in \mathcal{A}$ is said to be in the class $k-U C V, k \geq 0$, if and only if,

$$
\frac{\left(z f^{\prime}(z)\right)^{\prime}}{f^{\prime}(z)} \prec p_{k}(z), \quad(z \in U) .
$$

The classes $k-S T$ and $k-U C V$ were further generalized by Shams et al, [11], to the $K D(k, \beta)$ and $S D(k, \beta)$, respectively, with respect to the conic domain $G(k, \beta), k \geq 0$ and $0 \leq \beta<1$ which is

$$
G(k, \beta)=\{w: \mathfrak{R e} w>k|w-1|+\beta\} .
$$

Now using the concepts of Janowski functions and the conic regions, we define the following class of functions.

Definition 1.5. A function $p(z)$ is said to be in the class $k-P[A, B], k \geq 0,-1 \leq B<$ $A \leq 1$, if and only if

$$
p(z) \prec \frac{(A+1) p_{k}(z)-(A-1)}{(B+1) p_{k}(z)-(B-1)}, \quad(z \in U),
$$

where $p_{k}(z)$ is defined in $(1.8)$.

Geometrically, the function $p(z) \in k-P[A, B]$, takes all values from the domain $\Omega_{k}[A, B],-1 \leq B<A \leq 1, k \geq 0$ which is defined as

$$
\Omega_{k}[A, B]=\left\{w: \mathfrak{R e}\left(\frac{(B-1) w(z)-(A-1)}{(B+1) w(z)-(A+1)}\right)>k\left|\frac{(B-1) w(z)-(A-1)}{(B+1) w(z)-(A+1)}-1\right|\right\},
$$


or equivalently as

$$
\Omega_{k}[A, B]=\left\{\begin{array}{c}
u+i v:\left[\left(B^{2}-1\right)\left(u^{2}+v^{2}\right)-2(A B-1) u+\left(A^{2}-1\right)\right]^{2} \\
>k^{2}\left[\begin{array}{c}
\left(-2(B+1)\left(u^{2}+v^{2}\right)+2(A+B+2) u-2(A+1)\right)^{2} \\
+4(A-B)^{2} v^{2}
\end{array}\right]
\end{array}\right\} .
$$

The domain $\Omega_{k}[A, B]$ retains the conic domain $\Omega_{k}$ inside the circular region defined by $\Omega[A, B]$. The impact of $\Omega[A, B]$, on the conic domain $\Omega_{k}$, changes the original shape of the conic regions. The ends of hyperbola and parabola gets closer to one another but never meet anywhere and the ellipse gets the oval shape. When $A \rightarrow 1, B \rightarrow-1$ the radius of the circular disk define by $\Omega[A, B]$ tends to infinity, consequently the arm of the hyperbola and parabola expand to the oval turns into ellipse. We see that $\Omega_{k}[1,-1]=\Omega_{k}$, the conic domain defined by Kanas and Wiśniowska [4].

Definition 1.6. A function $f(z) \in \mathcal{A}$ is said to be in the class $k-S_{s}^{(t)} T[A, B], k \geq$ $0,-1 \leq B<A \leq 1$, if and only if

$$
\mathfrak{R e}\left(\frac{(B-1) \frac{z f^{\prime}(z)}{f_{t}(z)}-(A-1)}{(B+1) \frac{z f^{\prime}(z)}{f_{t}(z)}-(A+1)}\right)>k\left|\frac{(B-1) \frac{z f^{\prime}(z)}{f_{t}(z)}-(A-1)}{(B+1) \frac{z f^{\prime}(z)}{f_{t}(z)}-(A+1)}-1\right|, \quad(z \in U)
$$

or equivalently

where $f_{t}(z)$ is defined by $(1.3)$.

$$
\frac{z f^{\prime}(z)}{f_{t}(z)} \in k-P[A, B]
$$

In some special cases we have the well known classes presented and studied earlier:

(i) $k-S_{s}^{(1)} T[A, B]=k-S T[A, B],[8]$.

(ii) $0-S_{s}^{(t)} T[A, B]=S_{s}^{(t)}[A, B],[6]$.

(iii) $k-S_{s}^{(1)} T[1,-1]=k-S T,[5]$.

(iv) $k-S_{s}^{(1)} T[1-2 \beta,-1]=S D(k, \beta),[11]$.

(v) $0-S_{s}^{(1)} T[A, B]=S^{*}[A, B],[3]$

Definition 1.7. A function $f(z) \in \mathcal{A}$ is said to be in the class $k-U C_{s}^{(t)} V[A, B], k \geq$ $0,-1 \leq B<A \leq 1$, if and only if

$$
\mathfrak{R e}\left\{\frac{(B-1) \frac{\left(z f^{\prime}(z)\right)^{\prime}}{f_{t}^{\prime}(z)}-(A-1)}{(B+1) \frac{\left(z f^{\prime}(z)\right)^{\prime}}{f_{t}^{\prime}(z)}-(A+1)}\right\}>k\left|\frac{(B-1) \frac{\left(z f^{\prime}(z)\right)^{\prime}}{f_{t}^{\prime}(z)}-(A-1)}{(B+1) \frac{\left(z f^{\prime}(z)\right)^{\prime}}{f_{t}^{\prime}(z)}-(A+1)}-1\right|, \quad(z \in U)
$$

or equivalently

$$
\frac{\left(z f^{\prime}(z)\right)^{\prime}}{f_{t}^{\prime}(z)} \in k-P[A, B]
$$

where $f_{t}(z)$ is defined by $(1.3)$.

In some special cases we have the well known classes presented and studied earlier:

(i) $k-U C_{s}^{(1)} V[A, B]=k-U C V[A, B],[8]$.

(ii) $k-U C_{s}^{(1)} V[1,-1]=k-U C V,[4]$.

(iii) $k-U C_{s}^{(1)} V[A, B]=K D(k, \beta),[11]$.

(iv) $0-U C_{s}^{(1)} V[A, B]=C[A, B],[3]$.

It is easy to see that

$$
f(z) \in k-U C_{s}^{(t)} V[A, B] \Longleftrightarrow z f^{\prime}(z) \in k-S_{s}^{(t)} T[A, B] .
$$




\section{Main results}

\subsection{Integral representation}

First we give two meaningful conclusions about the classes $k-S_{s}^{(t)} T[A, B]$ and $k-$ $U C_{s}^{(t)} V[A, B]$.

Theorem 2.1. Let $f(z) \in k-S_{s}^{(t)} T[A, B]$. Then $f_{t}(z) \in k-S^{(1)} T[A, B] \in \mathcal{S}$.

Proof. For $f(z) \in k-S_{s}^{(t)} T[A, B]$, we can obtain

$$
\frac{z f^{\prime}(z)}{f_{t}(z)} \prec \frac{(A+1) p_{k}(z)-(A-1)}{(B+1) p_{k}(z)-(B-1)}, \quad(z \in U) .
$$

Substituting $z$ by $\epsilon^{\mu} z$ respectively $(\mu=0,1,2,3, \ldots, t-1)$, we have

$$
\frac{\epsilon^{\mu} z f^{\prime}\left(\epsilon^{\mu} z\right)}{f_{t}\left(\epsilon^{\mu} z\right)} \prec \frac{(A+1) p_{k}\left(\epsilon^{\mu} z\right)-(A-1)}{(B+1) p_{k}\left(\epsilon^{\mu} z\right)-(B-1)} \prec \frac{(A+1) p_{k}(z)-(A-1)}{(B+1) p_{k}(z)-(B-1)}, \quad(z \in U) .
$$

By definition of $f_{t}(z)$ and $\epsilon=\exp \left(\frac{2 \pi i}{t}\right)$, we know $\epsilon^{-\mu} f_{t}\left(\epsilon^{\mu} z\right)=f_{t}(z)$. Then equation (2.2) becomes

$$
\frac{z f^{\prime}\left(\epsilon^{\mu} z\right)}{f_{t}(z)} \prec \frac{(A+1) p_{k}(z)-(A-1)}{(B+1) p_{k}(z)-(B-1)}, \quad(z \in U)
$$

Let $(\mu=0,1,2,3, \ldots, t-1)$ in $(2.3)$, respectively. Making the convex combination of them, we can get

$$
\frac{z f_{t}^{\prime}(z)}{f_{t}(z)}=\frac{1}{t} \sum_{\mu=0}^{t-1} \frac{z f^{\prime}\left(\epsilon^{\mu} z\right)}{f_{t}(z)} \prec \frac{(A+1) p_{k}(z)-(A-1)}{(B+1) p_{k}(z)-(B-1)}, \quad(z \in U),
$$

because the function on right-hand site of (2.3) is convex univalent. That is, $f_{t}(z) \in$ $k-S_{s}^{(1)} T[A, B]=k-S T[A, B] \subset \mathcal{S},[8]$.

Putting $k=0$ in Theorem 2.1, we can obtain Corollary 2.2, below which is comparable to the result obtained by Kwon and Sim [6] .

Corollary 2.2. Let $f(z) \in S_{s}^{(t)} T[A, B]$. Then $f_{t}(z) \in S^{*}[A, B] \subset S$.

Theorem 2.3. Let $f(z) \in k-U C_{s}^{(t)} V[A, B]$. Then $f_{t}(z) \in U C V[A, B] \subset S$.

Proof. The proof of Theorem 2.3 is similar to that of Theorem 2.1 so the details are omitted.

Now we give the integral representations of the functions belonging to the classes $k-$ $S_{s}^{(t)} T[A, B]$ and $k-U C_{s}^{(t)} V[A, B]$.

Theorem 2.4. Let $f(z) \in k-S_{s}^{(t)} T[A, B]$. Then

$$
f_{t}(z)=z \cdot\left\{\exp (A-B) \frac{1}{t} \sum_{\mu=0}^{t-1} \int_{0}^{\epsilon^{\mu} z} \frac{\left(p_{k}(w(\varsigma))-1\right)}{t(B+1) p_{k}(w(\varsigma))-(B-1)} \mathrm{d} \varsigma\right\}
$$

where $\omega(z)$ is analytic in $U, \omega(0)=0$ and $|\omega(z)|<1$.

Proof. For $f(z) \in k-S_{s}^{(t)} T[A, B]$, from definition of the subordination we can have

$$
\frac{z f^{\prime}(z)}{f_{t}(z)}=\frac{(A+1) p_{k}(w(z))-(A-1)}{(B+1) p_{k}(w(z))-(B-1)}, \quad(z \in U)
$$


where $w(z)$ analytic in $U$, with $w(0)=0$ and $|w(z)|<1$. Substituting $z$ by $\epsilon^{\mu} z$ respectively $(\mu=0,1,2,3, \ldots, t-1)$, we have

$$
\frac{z f^{\prime}\left(\epsilon^{\mu} z\right)}{\epsilon^{-\mu} f_{t}\left(\epsilon^{\mu} z\right)}=\frac{(A+1) p_{k}\left(w\left(\epsilon^{\mu} z\right)\right)-(A-1)}{(B+1) p_{k}\left(w\left(\epsilon^{\mu} z\right)\right)-(B-1)} .
$$

For $(\mu=0,1,2,3, \ldots, t-1)$ and $z \in U$. Using the equalities (1.6) and (1.7) we have

$$
\frac{z f_{t}^{\prime}(z)}{f_{t}(z)}=\frac{1}{t} \sum_{\mu=0}^{t-1} \frac{(A+1) p_{k}\left(w\left(\epsilon^{\mu} z\right)\right)-(A-1)}{(B+1) p_{k}\left(w\left(\epsilon^{\mu} z\right)\right)-(B-1)}
$$

or equivalently,

$$
\frac{f_{t}^{\prime}(z)}{f_{t}(z)}-\frac{1}{z}=\frac{1}{t} \sum_{\mu=0}^{t-1} \frac{(A-B)\left(p_{k}\left(w\left(\epsilon^{\mu} z\right)\right)-1\right)}{z\left((B+1) p_{k}\left(w\left(\epsilon^{\mu} z\right)\right)-(B-1)\right)} .
$$

Integrating equality (2.8), we have

$$
\begin{aligned}
\log \frac{f_{t}(z)}{z} & =(A-B) \frac{1}{t} \sum_{\mu=0}^{t-1} \int_{0}^{z} \frac{\left(p_{k}\left(w\left(\epsilon^{\mu} \varsigma\right)\right)-1\right)}{\zeta\left((B+1) p_{k}\left(w\left(\epsilon^{\mu} \varsigma\right)\right)-(B-1)\right)} \mathrm{d} \varsigma \\
& =(A-B) \frac{1}{t} \sum_{\mu=0}^{t-1} \int_{0}^{\epsilon^{\mu} z} \frac{\left(p_{k}(w(\varsigma))-1\right)}{t(B+1) p_{k}(w(\varsigma))-(B-1)} \mathrm{d} \varsigma .
\end{aligned}
$$

Therefore arranging equality $(2.9)$ for $f_{t}(z)$, we can obtain

$$
f_{t}(z)=z \cdot\left\{\exp (A-B) \frac{1}{t} \sum_{\mu=0}^{t-1} \int_{0}^{\epsilon^{\mu} z} \frac{\left(p_{k}(w(\varsigma))-1\right)}{t(B+1) p_{k}(w(\varsigma))-(B-1)} \mathrm{d} \varsigma\right\},
$$

and so the proof of Theorem 2.4 is complete.

Putting $t=1$, in Theorem 2.4, we can obtain Corollary 2.5.

Corollary 2.5. Let $f(z) \in k-S T[A, B]$. Then

$$
f(z)=z \cdot\left\{\exp (A-B) \int_{0}^{z} \frac{\left(p_{k}(w(\varsigma))-1\right)}{t(B+1) p_{k}(w(\varsigma))-(B-1)} \mathrm{d} \varsigma\right\},
$$

where $\omega(z)$ is analytic in $U, \omega(0)=0$ and $|\omega(z)|<1$.

Putting $k=0$, in Theorem 2.4, we can obtain Corollary 2.6, below which is comparable to the result obtained by Kwon and Sim [6].

Corollary 2.6. Let $f(z) \in k-S_{s}^{(t)}[A, B]$. Then

$$
f_{t}(z)=z \cdot\left\{\exp (A-B) \frac{1}{t} \sum_{\mu=0}^{t-1} \int_{0}^{z} \frac{w(\varsigma)}{t(1+B w(\varsigma))} \mathrm{d} \varsigma\right\}
$$

where $\omega(z)$ is analytic in $U, \omega(0)=0$ and $|\omega(z)|<1$.

Putting $t=1, A=1$ and $B=-1$ in Theorem 2.4, we can obtain Corollary 2.7.

Corollary 2.7. Let $f(z) \in k-U S$. Then

$$
f(z)=z \cdot\left\{\exp \int_{0}^{z}\left(p_{k}(w(\varsigma))-1\right) \mathrm{d} \varsigma\right\}
$$

where $\omega(z)$ is analytic in $U, \omega(0)=0$ and $|\omega(z)|<1$. 
Theorem 2.8. Let $f(z) \in k-U C_{s}^{(t)} V[A, B]$. Then

$$
f_{t}(z)=\int_{0}^{z} \exp \left((A-B) \frac{1}{t} \sum_{\mu=0}^{t-1} \int_{0}^{\epsilon^{\mu} \varsigma} \frac{\left(p_{k}(w(\tau))-1\right)}{t(B+1) p_{k}(w(\tau))-(B-1)} \mathrm{d} \tau\right) d \varsigma,
$$

where $\omega(z)$ is analytic in $U, \omega(0)=0$ and $|\omega(z)|<1$.

Proof. The proof of Theorem 2.8 is similar to that of Theorem 2.4 so the details are omitted.

Theorem 2.9. Let $f(z) \in k-S_{s}^{(t)} T[A, B]$. Then

$$
\begin{aligned}
f(z)= & \int_{0}^{z} \exp \left((A-B) \frac{1}{t} \sum_{\mu=0}^{t-1} \int_{0}^{\epsilon^{\mu} \zeta} \frac{\left(p_{k}(w(\tau))-1\right)}{t(B+1) p_{k}(w(\tau))-(B-1)} \mathrm{d} \tau\right) \\
& \times\left(\frac{(A+1) p_{k}(w(\varsigma))-(A-1)}{(B+1) p_{k}(w(\varsigma))-(B-1)}\right) d \varsigma,
\end{aligned}
$$

where $\omega(z)$ is analytic in $U, \omega(0)=0$ and $|\omega(z)|<1$.

Proof. Let $f(z) \in k-S_{s}^{(t)} T[A, B]$, then from equalities (2.4) and (2.5) we have

$$
\begin{aligned}
f^{\prime}(z)= & \frac{f_{t}(z)}{z}\left(\frac{(A+1) p_{k}(w(z))-(A-1)}{(B+1) p_{k}(w(z))-(B-1)}\right) \\
= & \exp (A-B) \frac{1}{t} \sum_{\mu=0}^{t-1} \int_{0}^{\epsilon^{\mu} z} \frac{\left(p_{k}(w(\tau))-1\right)}{t(B+1) p_{k}(w(\tau))-(B-1)} \mathrm{d} \tau \\
& \times\left(\frac{(A+1) p_{k}(w(z))-(A-1)}{(B+1) p_{k}(w(z))-(B-1)}\right) .
\end{aligned}
$$

Integrating the equality $(2.10)$, we have

$$
\begin{aligned}
f(z)= & \int_{0}^{z} \exp \left((A-B) \frac{1}{t} \sum_{\mu=0}^{t-1} \int_{0}^{\epsilon^{\mu} \varsigma} \frac{\left(p_{k}(w(\tau))-1\right)}{t(B+1) p_{k}(w(\tau))-(B-1)} \mathrm{d} \tau\right) \\
& \times\left(\frac{(A+1) p_{k}(w(\varsigma))-(A-1)}{(B+1) p_{k}(w(\varsigma))-(B-1)}\right) \mathrm{d} \varsigma .
\end{aligned}
$$

and so the proof of Theorem 2.9 is complete.

Putting $k=0$, in Theorem 2.9, we can obtain Corollary 2.10, below which is comparable to the result obtained by Kwon and Sim [6].

Corollary 2.10. Let $f(z) \in S_{s}^{(t)} T[A, B]$. Then

$$
f(z)=\int_{0}^{z} \exp \left((A-B) \frac{1}{t} \sum_{\mu=0}^{t-1} \int_{0}^{\epsilon^{\mu} \zeta} \frac{w(\tau)}{\tau(1+B w(\tau))} \mathrm{d} \tau\right)\left(\frac{1+A w(\varsigma)}{1+B w(\varsigma)}\right) d \varsigma .
$$

where $\omega(z)$ is analytic in $U, \omega(0)=0$ and $|\omega(z)|<1$.

By applying similar method as in Theorem 2.9, we have

Theorem 2.11. Let $f(z) \in k-U C_{s}^{(t)} V[A, B]$. Then

$$
\begin{aligned}
f(z)= & \int_{0}^{z} \frac{1}{\xi} \int_{0}^{\xi} \exp \left((A-B) \frac{1}{t} \sum_{\mu=0}^{t-1} \int_{0}^{\epsilon^{\mu} \varsigma} \frac{\left(p_{k}(w(\tau))-1\right)}{t(B+1) p_{k}(w(\tau))-(B-1)} \mathrm{d} \tau\right) \\
& \times\left(\frac{(A+1) p_{k}(w(\varsigma))-(A-1)}{(B+1) p_{k}(w(\varsigma))-(B-1)}\right) \mathrm{d} \varsigma \mathrm{d} \xi,
\end{aligned}
$$

where $\omega(z)$ is analytic in $U, \omega(0)=0$ and $|\omega(z)|<1$. 


\subsection{Convolution conditions}

In this sections, we provide the convolutions conditions for the classes $k-S_{s}^{(t)} T[A, B]$ and $k-U C_{s}^{(t)} V[A, B]$.

Theorem 2.12. A function $f(z) \in k-S_{s}^{(t)} T[A, B]$, if and only if

$$
\begin{aligned}
& \frac{1}{z}\left\{f ( z ) * \left(\frac{z}{(1-z)^{2}}\left((B+1) p_{k}\left(e^{i \theta}\right)-(B-1)\right)\right.\right. \\
& \left.\left.-\left((A+1) p_{k}\left(e^{i \theta}\right)-(A-1)\right) h(z)\right)\right\} \neq 0,
\end{aligned}
$$

for all $z \in U$ and $0 \leq \theta<2 \pi$. The coefficients of $h(z)=z+b_{2} z^{2}+\cdots$ are given by (1.5). The function $p_{k}(z)$ is defined in (1.8).

Proof. Assume that $f(z) \in k-S_{s}^{(t)} T[A, B]$, then we have

$$
\frac{z f^{\prime}(z)}{f_{t}(z)} \prec \frac{(A+1) p_{k}(z)-(A-1)}{(B+1) p_{k}(z)-(B-1)}, \quad(z \in U)
$$

if and only if

$$
\frac{z f^{\prime}(z)}{f_{t}(z)} \neq \frac{(A+1) p_{k}\left(e^{i \theta}\right)-(A-1)}{(B+1) p_{k}\left(e^{i \theta}\right)-(B-1)},
$$

for all $z \in U$, and $0 \leq \theta<2 \pi$. The condition (2.12), can be written as

$$
\frac{1}{z}\left\{z f^{\prime}(z)\left[(B+1) p_{k}\left(e^{i \theta}\right)-(B-1)\right]-f_{t}(z)\left[\left((A+1) p_{k}\left(e^{i \theta}\right)-(A-1)\right)\right]\right\} \neq 0 .
$$

On the other hand it is well known that

$$
z f^{\prime}(z)=f(z) * \frac{z}{(1-z)^{2}} .
$$

And from (1.4), we have

$$
f_{t}(z)=z+\sum_{n=2}^{\infty} a_{n} b_{n} z^{n}=(f * h)(z)
$$

where

$$
h(z)=z+\sum_{n=2}^{\infty} b_{n} z^{n}
$$

and where $b_{n}$ is given by (1.5). Substituting (2.14) and (2.15) in (2.13), we can get (2.11). This completes the proof of the Theorem 2.12.

Putting $t=1$, in Theorem 2.12, we can obtain Corollary 2.13.

Corollary 2.13. A function $f(z) \in k-S T[A, B]$, if and only if

$$
\frac{1}{z}\left\{f(z) *\left(\frac{z}{(1-z)^{2}}\left(1+B\left(e^{i \theta}\right)\right)-\frac{z}{(1-z)}\left(1+A\left(e^{i \theta}\right)\right)\right)\right\} \neq 0,
$$

for all $z \in U$.

Putting $k=0$, in Theorem 2.12, we can obtain Corollary 2.14.

Corollary 2.14. A function $f(z) \in S_{s}^{(t)}[A, B]$, if and only if

$$
\frac{1}{z}\left\{f(z) *\left(\frac{z}{(1-z)^{2}}\left(1+B\left(e^{i \theta}\right)\right)-h(z)\left(1+A\left(e^{i \theta}\right)\right)\right)\right\} \neq 0,
$$

for all $z \in U$ and $0 \leq \theta<2 \pi$, where $h(z)$ is given by (2.16). 
Theorem 2.15. For $k \geq 0,-1 \leq B<A \leq 1$. A function $f(z) \in k-U C_{s}^{(t)} V[A, B]$, if and only if

$$
\frac{1}{z}\left\{f(z) *\left(\frac{z(B+1) p_{k}\left(e^{i \theta}\right)-(B-1)}{(1-z)^{2}}-\left((A+1) p_{k}\left(e^{i \theta}\right)-(A-1)\right) h(z)\right)^{\prime}\right\} \neq 0,
$$

where $z \in U, 0 \leq \theta<2 \pi$.

Proof. The proof of Theorem 2.15, is similar to that of Theorem 2.12 , so the detail are omitted.

\subsection{Coefficient inequalities}

Finally, we provide some coefficient inequalities which are sufficient for a function to be in the class $k-S_{s}^{(t)} T[A, B]$ or to be in the class $k-U C_{s}^{(t)} V[A, B]$.

Theorem 2.16. Assume that $t$ is a positive integer, $-1 \leq B<A \leq 1$ and $k \geq 0$. If a function $f(z) \in A$ of the form (1.1) satisfies the condition

$$
\begin{aligned}
& \sum_{n=1}^{\infty} 2(k+1) \operatorname{tn}+|(\operatorname{tn}(B+1)+(B-A))|\left|a_{t n+1}\right| \\
+ & \sum_{n=2, n \neq l t+1}^{\infty} 2(k+1) n+|(n(B+1))|\left|a_{n}\right|<|B-A|,
\end{aligned}
$$

then $f(z)$ is in the class $k-S_{s}^{(t)} T[A, B]$.

Proof. Assume that (2.17) holds, then it suffices to show that

$$
k\left|\frac{(B-1) \frac{z f^{\prime}(z)}{f_{t}(z)}-(A-1)}{(B+1) \frac{z f^{\prime}(z)}{f_{t}(z)}-(A+1)}-1\right|-\mathfrak{R e}\left[\frac{(B-1) \frac{z f^{\prime}(z)}{f_{t}(z)}-(A-1)}{(B+1) \frac{z f^{\prime}(z)}{f_{t}(z)}-(A+1)}-1\right]<1 .
$$

We have

$$
\begin{aligned}
& k\left|\frac{(B-1) \frac{z f^{\prime}(z)}{f_{t}(z)}-(A-1)}{(B+1) \frac{z f^{\prime}(z)}{f_{t}(z)}-(A+1)}-1\right|-\mathfrak{R e}\left[\frac{(B-1) \frac{z f^{\prime}(z)}{f_{t}(z)}-(A-1)}{(B+1) \frac{z f^{\prime}(z)}{f_{t}(z)}-(A+1)}-1\right] \\
\leq & (k+1)\left|\frac{(B-1) z f^{\prime}(z)-(A-1) f_{t}(z)}{(B+1) z f^{\prime}(z)-(A+1) f_{t}(z)}-1\right| \\
= & 2(k+1)\left|\frac{f_{t}(z)-z f^{\prime}(z)}{(B+1) z f^{\prime}(z)-(A+1) f_{t}(z)}\right| \\
\leq & 2(k+1) \frac{\sum_{n=2}^{\infty}\left|b_{n}-n\right|\left|a_{n}\right|}{|B-A|-\sum_{n=2}^{\infty}\left|n(B+1)-(A+1) b_{n}\right|\left|a_{n}\right|} .
\end{aligned}
$$

The last expression is bounded by 1 , if

$$
\sum_{n=2}^{\infty}\left(2(k+1)\left(n-b_{n}\right)+\left|n(B+1)-(A+1) b_{n}\right|\right)\left|a_{n}\right|<|B-A| .
$$

Using (1.5) in (2.18) we have

$$
\begin{aligned}
& \sum_{n=1}^{\infty} 2(k+1) t n+|(\operatorname{tn}(B+1)+(B-A))|\left|a_{t n+1}\right| \\
+ & \sum_{n=2, n \neq l t+1}^{\infty} 2(k+1) n+|(n(B+1))|\left|a_{n}\right|<|B-A|,
\end{aligned}
$$

and this completes the proof of Theorem 2.16. 
Putting $t=1$, in Theorem 2.16, we can obtain the following Corollary 2.17, which is comparable to a result obtained by Noor and Malik in [8].

Corollary 2.17. A function $f(z) \in \mathcal{A}$ of the form (1.1) is in the class $k-S T[A, B]$, if it satisfies the condition

$$
\sum_{n=2}^{\infty}\{2(k+1)(n-1)+|(n(B+1)+(A+1))|\}\left|a_{n}\right|<|B-A|,
$$

where $k \geq 0,-1 \leq B<A \leq 1$.

Putting $k=0$, in Theorem 2.16, we can obtain the following Corollary 2.18, which is comparable to a result obtained by Kwon and Sim in [6].

Corollary 2.18. A function $f(z) \in \mathcal{A}$ of the form (1.1) is in the class $S_{s}^{(t)} T[A, B]$, if it satisfies the condition

$$
\sum_{n=1}^{\infty}(t n+(A-B)(t n+1))\left|a_{t n+1}\right|+\sum_{n=2, n \neq l t+1}^{\infty}(1+|B|) n\left|a_{n}\right|<|B-A|,
$$

where $-1 \leq B<A \leq 1$.

Putting $t=1, A=1$ and $B=-1$ in Theorem 2.16, we can obtain Corollary 2.19, which is comparable to a result obtained by Kanas and Wiśniowska in [4].

Corollary 2.19. A function $f(z) \in \mathcal{A}$ of the form (1.1) is in the class $k-S T$, if it satisfies the condition

$$
\sum_{n=2}^{\infty}\{n+k(n-1)\}\left|a_{n}\right|<1, \quad k>0 .
$$

Putting $t=1, A=1-2 \alpha, B=-1$ with $0 \leq \beta<1$ in Theorem 2.16, we can obtain Corollary 2.20, which is comparable to a result obtained by Shams et al. in [11].

Corollary 2.20. A function $f(z) \in \mathcal{A}$ of the form (1.1) is in the class $S D(k, \beta)$, if it satisfies the condition

$$
\sum_{n=2}^{\infty}\{n(k+1)-(k+\beta)\}\left|a_{n}\right|<1-\beta,
$$

where $0 \leq \beta<1$. then $k \geq 0$.

Putting $t=1, A=1-2 \beta, B=-1$ with $0 \leq \beta<1$ and $k=0$ in Theorem 2.16, we can obtain Corollary 2.21, below which is comparable to the known result obtained by Silverman in [13].

Corollary 2.21. A function $f(z) \in \mathcal{A}$ of the form (1.1) is in the class $S^{*}(\beta)$, if it satisfies the conditions

where $0 \leq \beta<1$.

$$
\sum_{n=2}^{\infty}\{n-\beta\}\left|a_{n}\right|<1-\beta,
$$

Theorem 2.22. A function $f(z) \in \mathcal{A}$ and of the form (1.1), is in the class $k-U C_{s}^{(t)} V[A, B]$, if it satisfies the condition

$$
\begin{aligned}
& \sum_{n=2}^{\infty}[2(k+1) t n+|n t(B+1)+(B-A)|](t n+1)\left|a_{t n+1}\right| \\
& \quad+\sum_{n=2, n \neq l t+1}^{\infty}(2(k+1) n+n(B+1))\left|n a_{n}\right|<|B-A|,
\end{aligned}
$$

where $-1 \leq B<A \leq 1$ and $k \geq 0$. 
Proof. The proof of Theorem 2.22, is similar to that of Theorem 2.16, so the detail are omitted.

\section{References}

[1] M. Arif, K.I. Noor, and R. Khan, On subclasses of analytic functions with respect to symmetrical points, Abst. Appl. Anal. 2012, 790689, 2012.

[2] R. Chand and P. Singh, On certain schlicht mapping, Indian J. Pure App. Math. 10, 1167-1174, 1979.

[3] W. Janowski, Some extremal problem for certain families of analytic functions, Ann. Polon. Math. 28 (3), 298-326, 1973.

[4] S. Kanas and A. Wiśniowska, Conic regions and k-uniform convexity, J. Comput. Appl. Math. 105, 327-336, 1999.

[5] S. Kanas and A. Wiśniowska, Conic domains and starlike functions, Rev. Roumaine Math. Pure. Appl. 45 (4), 647-657, 2000.

[6] O. Kwon and Y. Sim, A certain subclass of Janowski type functions associated with k-symmetric points, Commun. Korean Math. Soc. 28, 143-154, 2013.

[7] S.S. Miller and P.T. Mocanu, Differential Subordinations, Theory and Applications, in: Series of Monographs and Textbooks in Pure and Applied Mathematics 225, Marcel Dekker Inc., New York, 2000.

[8] K.I. Noor and S.N. Malik, On coefficient inequalities of functions associated with conic domains, Comput. Math. Appl. 62, 2209-2217, 2011.

[9] K.I. Noor and S. Mustafa, Some classes of analytic functions related with functions of bounded radius rotation eith respect to symmetrical points, J. Math. Ineq. 2 (3), 267-276, 2009.

[10] K. Sakaguchi, On a certain univalent mapping, J. Math. Soc. Japan 11, 72-75, 1959.

[11] S. Shams, S.R. Kulkarni, and J.M. Jahangiri, Classes of uniformly starlike and convex functions, Int. J. Math. Math. Sci. 55, 2959-2961, 2004.

[12] T.N. Shanmugam, C. Ramachandran, and V. Ravichandran, Fekete-Szegö problem for subclass of starlike functions with respect to symmetric points, Bull. Korean Math. Soc. 43 (3), 589-598, 2006.

[13] H. Silverman, Univalent functions with negative coefficients, Proc. Amr. Math. Soc. 51, 109-116, 1975.

[14] J. Sokół, Some remarks on the class of functions starlike with respect to symmetric points, Folia Sci. Univ. Tech. Resov. 73, 79-91, 1990. 